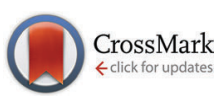

Cite this: Phys. Chem. Chem. Phys., 2016, 18, 15458

DOI: 10.1039/c6cp90132h

www.rsc.org/pccp

\section{Correction: Assembly and relaxation behaviours of phosphatidylethanolamine monolayers investigated by polarization and frequency resolved SFG-VS}

\author{
Feng Wei, ${ }^{a}$ Wei Xiong, ${ }^{a}$ Wenhui Li, ${ }^{a}$ Wangting Lu, ${ }^{a}$ Heather C. Allen ${ }^{b}$ and \\ Wanquan Zheng ${ }^{\text {ac }}$
}

Correction for 'Assembly and relaxation behaviours of phosphatidylethanolamine monolayers investigated by polarization and frequency resolved SFG-VS' by Feng Wei et al., Phys. Chem. Chem. Phys., 2015, 17, 25114-25122.

The authors would like to make some corrections to their article based on some recent calculations:

The spectral resolution of IR-scanning sum frequency generation vibrational spectroscopy (SFG-VS) system can be calculated using eqn (13) in ref. 1:

$$
\Delta \nu_{\mathrm{Instr}}^{2}=\frac{8 \ln (2)}{(2 \pi c)^{2}} \Delta \omega_{\mathrm{SF}^{2}}=\frac{8 \ln (2)}{(2 \pi c)^{2}}\left(\Delta \omega_{\mathrm{IR}}^{2}+\Delta \omega_{\mathrm{Vis}}^{2}\right)
$$

where $\Delta \omega_{\text {IR }}$ and $\Delta \omega_{\text {Vis }}$ are the Guassian bandwidths/linewidths of incident IR beam and visible beam, respectively.

So based on our latest experiments and calculation results:

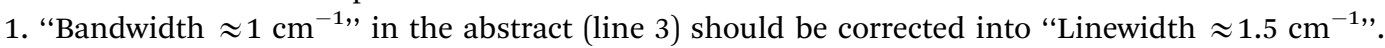

2. "The bandwidth of the final IR beam is calculated to be $\approx 1 \mathrm{~cm}^{-1}$, about twice the transform-limited width of a $30 \mathrm{ps}$ Gaussian pulse $\left(0.48 \mathrm{~cm}^{-1}\right)$." in "Experimental" (page 2, left column, last third line) should be corrected into "The linewidths of the final IR beams are calculated to be $\approx 1.5 \mathrm{~cm}^{-1}$."

3. "The spectral resolution of the current SFG system is $\approx 1 \mathrm{~cm}^{-1}$." in "Introduction" (page 2, left column, paragraph 2, line 11-12) should be corrected into "The calculated spectral resolution $\Delta \nu_{\text {Instr }}$ of the current SFG system is about $4.6 \mathrm{~cm}^{-1}$."

\title{
Reference
}

1 F. Wei, W. X. Xia, Z. J. Hu, W. H. Li, J. Y. Zhang and W. Q. Zheng, Chin. J. Chem. Phys., 2016, 29, $171-178$.

The Royal Society of Chemistry apologises for these errors and any consequent inconvenience to authors and readers.

\footnotetext{
${ }^{a}$ Institution for Interdisciplinary Research, Jianghan University, Building J13, Room C202, 8th Triangle Lake Road, Zhuankou District, Wuhan, Hubei, 430056, China. E-mail:weifeng@jhun.edu.cn

${ }^{b}$ Department of Chemistry and Biochemistry, The Ohio State University, 100 West 18th Avenue, Columbus, OH 43210, USA

${ }^{c}$ Institut des Sciences Moléculaires d'Orsay, Université de Paris-Sud, 91405, ORSAY Cedex, France
} 